\title{
Cosmology and the massive photon frequency shift in the Standard-Model Extension
}

\author{
Alessandro D. A. M. Spallicci ${ }^{1,2,3,4, a}$, José A. Helayël-Neto ${ }^{5, b}$, Martín López-Corredoira ${ }^{6,7, c}$, \\ Salvatore Capozziello ${ }^{8,9, \mathrm{~d}}$ \\ ${ }^{1}$ Observatoire des Sciences de l'Univers en région Centre (OSUC) UMS 3116, Université d'Orléans, 1A rue de la Férollerie, 45071 Orléans, \\ France \\ ${ }^{2}$ Pôle de Physique, Collegium Sciences et Techniques (CoST), Université d'Orléans, Rue de Chartres, 45100 Orléans, France \\ ${ }^{3}$ Laboratoire de Physique et Chimie de l'Environnement et de l'Espace (LPC2E) UMR 7328, Centre National de la Recherche Scientifique (CNRS), \\ Campus CNRS, 3A Avenue de la Recherche Scientifique, 45071 Orléans, France \\ ${ }^{4}$ Departamento de Física Teórica, Instituto de Física, Universidade do Estado do Rio de Janeiro (UERJ), Rua São Francisco Xavier 524, Maracanã, \\ RJ 20550-013 Rio de Janeiro, Brazil \\ ${ }^{5}$ Departamento de Astrofísica, Cosmologia e Interações Fundamentais (COSMO), Centro Brasileiro de Pesquisas Físicas (CBPF), Rua Xavier \\ Sigaud 150, Urca, RJ 22290-180 Rio de Janeiro, Brazil \\ ${ }^{6}$ Instituto de Astrofísica de Canarias (IAC), C/ Vía Láctea s/n, 38205 San Cristóbal de La Laguna, Tenerife, Spain \\ ${ }^{7}$ Departamento de Astrofísica, Sección de Física, Facultad de Ciencias, Universidad de La Laguna, Avenida Astrofísico Francisco Sánchez s/n, \\ Apartado 456, 38200 San Cristóbal de La Laguna, Tenerife, Spain \\ ${ }^{8}$ Dipartimento di Fisica Ettore Pancini, Università degli Studi di Napoli, Federico II (UNINA), Via Cinthia 9, 80126 Napoli, Italy \\ ${ }^{9}$ Laboratory for Theoretical Cosmology, Tomsk State University of Control Systems and Radioelectronics (TUSUR), 40 prospect Lenina, 634050 \\ Tomsk, Russia
}

Received: 22 May 2020 / Accepted: 24 November 2020 / Published online: 5 January 2021

(C) The Author(s) 2020

\begin{abstract}
The total red shift $z$ might be recast as a combination of the expansion red shift and a static shift due to the energy-momentum tensor non-conservation of a photon propagating through Electro-Magnetic (EM) fields. If massive, the photon may be described by the de Broglie-Proca (dBP) theory which satisfies the Lorentz(-Poincaré) Symmetry (LoSy) but not gauge-invariance. The latter is regained in the Standard-Model Extension (SME), associated with LoSy Violation (LSV) that naturally dresses photons of a mass. The non-conservation stems from the vacuum expectation value of the vector and tensor LSV fields. The final colour (red or blue) and size of the static shift depend on the orientations and strength of the LSV and EM multiple fields encountered along the path of the photon. Turning to cosmology, for a zero $\Omega_{\Lambda}$ energy density, the discrepancy between luminosity and red shift distances of SNeIa disappears thanks to the recasting of $z$. Massive photons induce an effective dark energy acting 'optically' but not dynamically.
\end{abstract}

\footnotetext{
a e-mail: spallicci@cnrs-orleans.fr (corresponding author)

be-mail: josehelayel@gmail.com

c e-mail: fuego.templado@gmail.com

de-mail: salvatore.capozziello@unina.it
}

\section{Introduction}

Astronomy is almost entirely built up from information coming from Electro-Magnetic (EM) signals, interpreted with Maxwellian linear and massless electromagnetism. This latter is possibly an approximation of a broader theory, as Newtonian gravitation is of the Einsteinian one. Observations [1-3] have led to the proposal that the universe contains up to $96 \%$ dark matter and dark energy (both entities thus far remaining theoretically unexplained and experimentally undetected) and holds to general relativity, as the correct theory of gravitation. Others, unconvinced by these ad hoc ingredients, propose new theories of gravitation, though general relativity scores high marks in all tests so far.

Faced with this dichotomy and the respective pitfalls, we turn to the signals. Could a different interpretation of light lead to a third option? After all, modern physics - relativity and quantum mechanics - started with a new interpretation of light. Ultimately, physics is in any case obliged to examine its foundations through either the formulation of new types of matter and energy or embracing new conceptions of gravitation or electromagnetism.

The photon is the only massless free particle of the successful Standard-Model (SM), lately challenged by the neutrino mass, the light mass of the Higgs boson and the absence 
of candidate particles for the dark universe. So let us focus on massive photons, leaving non-linear electromagnetism for future work.

The official photon mass upper limit is $10^{-54} \mathrm{~kg}$ [4], but, as pointed out in [5], such a limit [6-8] arises primarily from modelling rather than measurement.

In 1922 de Broglie proposed a massive photon [9] and, through the group velocity dispersion of the latter, estimated the mass upper limit as $10^{-53} \mathrm{~kg}[10,11]$. The origin of his conception of the photon mass resides in his theory of fusion, which couples two or more free Dirac fields to produce a scalar or vector Klein-Gordon equation, typically an electron-positron or neutrino-anti-neutrino duet [12-18]. In [18] the modified Maxwell equations were first written in a non-covariant form. Thus, de Broglie, and accidentally his disciple Proca [19-22], laid down the first massive electromagnetism, compliant with the Lorentz(-Poincaré) Symmetry (LoSy), though not gauge-invariant. Contrary to common belief, the Proca Lagrangian describes generic composite particles, among which Proca states that the photons are massless 'doublets de masse nulle' [22]. For a comprehensive monograph see [23].

Energy-momentum non-conservation already manifests itself in the Maxwellian theory for a photon crossing spacetime dependent electromagnetic (EM) background fields (host galaxy, intergalactic and Milky Way). Instead, we show here that the de Broglie-Proca ( $\mathrm{dBP}$ ) massive photon undergoes energy-momentum non-conservation also in the case of constant EM fields, since it couples to the non-constant EM background potential.

Motivated by the above challenges, the SM Extension (SME), based on the LoSy Violation (LSV), was put forward $[24,25]$. The LSV breaks the invariance of the laws of physics for all positions and inertial velocities of the observers, and thus naturally accommodates anisotropies. The SM is LoSy invariant up to the energy scales at which the LSV occurred in the early universe. The SME allows the testing of the lowenergy manifestations of the LSV in the present universe.

The foundations of the analysis of light propagation in the SME were laid down in [26,27] . Going beyond the SM, a massive photon emerges by scrutiny of the group velocity dispersion or by recasting the SME Lagrangian. The effective, and frame-dependent, mass is proportional to the value of the LSV parameters and, in contrast to the dBP formalism, the SME massive photon is gauge-invariant [26,27] and is compatible with the LSV upper limits [28,29]. Some of the benefits and drawbacks of attributing a mass to photons have been analysed recently [30-34].

Moreover, in specific conditions sub- and super-luminal velocities, imaginary and complex frequencies, and birefringence appear. Evidently in any condition, LSV anisotropy and inhomogeneity are present.
Also in the SME, the photon energy-momentum tensor is not conserved in vacuum, and most remarkably, includes terms depending on constant LSV and EM fields [27]. Furthermore, we have determined a non-expansion related frequency shift (towards the red or the blue) zLSV [35].

Quantified by the Hubble(-Humason)-Lemaître constant $H_{0}$ and detected at the end of the '20s, the cosmological expansion stretches wavelengths from astrophysical sources, including Type Ia supernovae (SNeIa). But since the end of the '90s, these latter, considering their faded luminosity with respect to the expected energy emitted, prompted the conception of dark energy, a mysterious fluid acting as the source of the cosmic accelerated expansion [36,37].

If the cosmic flow expanded at a constant rate, an SNIa red shift would be directly proportional to its distance, and thus to its brightness. Instead, the accelerating universe filled with dark energy implies that space expanded less quickly in the past than it does now. The light from distant objects such as SNeIa is less stretched during its journey to us, given how slowly space expanded during much of the time. An SNIa at a given distance (computed through its brightness, since SNeIa are believed to be standard candles) appears less red shifted than it would in a universe without dark energy. To put it another way, the red shift is not a measurement of distance but of expansion. When a distant source is observed, the lower expansion induces a lower red shift.

The apparent acceleration of the younger universe could be simply accounted for by a non-zero cosmological constant (originally proposed by Einstein to refute an expanding universe) on the left (geometrical) side of the field equations, or else by an extra term on the right (energy-matter) side acting as a source term for gravity, and corresponding to a vacuum energy density.

Various hypotheses were investigated concerning the nature of dark energy, e.g. [38,39], among which was the extension of the gravity sector [40-43].

Somewhat similarly, in this paper we also refer to an extension, namely that of the SM. Indeed, we identify the vacuum energy density in the framework of the SME as being due to the LSV field, which manifests itself through the $z_{\text {LSV }}$ shift.

The above-mentioned third option may be checked by adding algebraically the $z_{\text {LSV }}$ shift to the cosmological expansion shift $z_{\mathrm{C}}$ in the analysis of the data from SNeIa. We go through Baryonic Acoustic Oscillation (BAO), Cosmic Microwave Background (CMB) and gravitational lensing, often reputed to back the existence of dark energy, to see if any counter-evidence would threaten our hypothesis. We also deal with time dilation in SNeIa.

Attributing different origins to the nature of dark energy does not fully describe the scope of the debate that arose since the appearance of $[36,37]$. One school of criticism focuses on the reliability of SNeIa, BAO, CMB and gravitational 
lensing data, and challenges the counterarguments defending the evidence for dark energy based on data, e.g., [44-48].

Another school of thought addresses alternatives to dark energy, of which we mention a few: photons oscillating into axions to produce a dimming effect for SNeIa $[49,50]$, the different spectrum of primordial fluctuations generated by inflation [51], timescape cosmology and the inhomogeneous universe [52], SNIa luminosity depending on red shift [53, 54], stronger evidence of acceleration dipole approximately aligned with the CMB dipole than evidence of a monopole dark energy acceleration $[55,56]$.

Last but not least, the identification of SNeIa as standard candles is at risk of being breached, e.g., [57].

We wish to emphasise that we do not take a position on data reliability. Our main point is to recast the red shift, nowadays uniquely explained by expansion; our recasting may be tailored to whatever set of data on which consensus will be reached. Moreover, a lesser $z_{\text {LSV }}$ shift value could coexist with dark energy.

Generally speaking, the Concordance Lambda-Cold Dark Matter $(\Lambda \mathrm{CDM})$ model may not rest on very firm ground, if Planck satellite CMB data, commonly reputedly its pillar, are reread and significantly support a closed universe [58].

In conclusion, our work is part of a large and scientifically healthy afflatus that revisits modern cosmology to verify its soundness.

Some wording on the relations between LSV, SME, extensions of the SM and photon mass are in order. LSV is not a sufficient condition to induce an effective mass. Generally speaking, extension of the SM such as the Minimally Supersymmetrised SM (MSSM), the Massive Neutrino Model (MNM), the Grand Unification Theory (GUT) do not induce an effective mass either. Thereby, it is the SME which is based on LSV that may cause an effective mass. Let us be more specific.

The SME-LSV factors are represented by a $k_{\alpha}^{\mathrm{AF}}\left[\right.$ metre $\left.^{-1}\right]$ 4-vector when the handedness of the Charge conjugationParity-Time reversal (CPT) symmetry is odd and by a $k_{\mathrm{F}}^{\alpha \nu \rho \sigma}$ [dimensionless] tensor when even. The $k_{\alpha}^{\mathrm{AF}}$ vector coming from the Carroll-Field-Jackiw Lagrangian [59] induces always a mass, while the $k_{\mathrm{F}}^{\alpha \nu \rho \sigma}$ tensor only in a supersymmetrised context after photino integration [26,27].

The SME is built up by means of the Effective Field Theory approach and has the SM and General Relativity (GR) as possible limiting cases. In this paper, we work within a scenario such that the LSV does not leave its imprints in the GR sector. We instead focus on a special situation where the LSV is present in the photonic sector, through the $k_{\alpha}^{\mathrm{AF}}$ and the $k_{\mathrm{F}}^{\alpha \nu \rho \sigma}$ terms. The space-time metric, spin connection and curvature are unaffected by the LSV; we actually maintain Minkowski space-time with the anisotropies parametrised by $k^{\mathrm{AF}}$ and $k^{\mathrm{F}}$. This means that the effects of the LSV on the red shift stem exclusively from the presence of the violat- ing parameters in the photonic sector. This is the viewpoint we adopt here. It is, however, a relevant matter, for future work to consider the presence of LSV in the gravitational sector [60-65] of SME and thereby reassess its effect on the red shift.

The rest of this paper is structured as follows. Sect. 2 shows the energy-momentum non-conservation of the $\mathrm{dBP}$ photon and prepares for the more difficult analysis in the SME dealt with in Sect. 3, devoted to light propagation in this context; Sect. 4 focuses on the reinterpretation of dark energy through the $z_{\text {LSV }}$ shift; Sects. 5 and 6 discuss the results and their implications.

Our metric has a $(+,-,-,-)$ signature; the Greek (Latin) indices run over $0,1,2,3(1,2,3)$. We adopt SI units.

\section{Energy non-conservation in Maxwell and de Broglie-Proca theories}

We recall here a basic feature of the $\mathrm{dBP}$ theory concerning the non-conservation of the photon energy-momentum and pave the way to the analysis in the SME. We imagine the photon crossing an electromagnetic background.

The dBP equations of motion derived from the Lagrangian [19] correspond to the divergence of the electric field and the curl of the magnetic field. They are given by

$\partial_{\alpha} F_{\mathrm{T}}^{\alpha \beta}+\mathcal{M}^{2} A_{\mathrm{T}}^{\beta}=\mu_{0} j^{\beta}$,

where $\mathcal{M}=\frac{m_{\gamma} c}{\hbar}, m_{\gamma}$ is the photon mass, $c=2.998 \times 10^{8}$ $\left[\mathrm{m} \mathrm{s}^{-1}\right]$ is the speed of light and $h=1.055 \times 10^{-34}[\mathrm{~kg}$ $\left.\mathrm{m}^{2} \mathrm{~s}^{-1}\right]$ is the reduced Planck constant. T stands for the total EM quantities due to background and photon contributions; $j^{\beta}$ is an external current, if it exists, and $\mu_{0}=1.257 \times 10^{-6}$ $\left[\mathrm{kg} \mathrm{m} \mathrm{A} \mathrm{m}^{-2} \mathrm{~s}^{-2}\right]$ is the magnetic permeability. Splitting the EM tensor field and the EM 4-potential in the background (capital letters) and photon (small letters) contributions, we have

$A_{\mathrm{T}}^{\beta}=A^{\beta}+a^{\beta} \quad F_{\mathrm{T}}^{\alpha \beta}=F^{\alpha \beta}+f^{\alpha \beta}$,

which substituted into Eq. (1) gives

$\partial_{\alpha} f^{\alpha \beta}+\mathcal{M}^{2} a^{\beta}=\mu_{0} j^{\beta}-\partial_{\alpha} F^{\alpha \beta}-\mathcal{M}^{2} A^{\beta}$.

Equation (3) tells us that the $\mathrm{dBP}$ photon interacts with the background through the potential even when the background field is constant. Indeed, if a field is constant, its associated potential is not

$F^{\alpha \beta}=\partial^{\alpha} A^{\beta}-\partial^{\beta} A^{\alpha}$.

Conversely, this is not the case for the Maxwell photon, Eq. (5), which interacts only with a non-constant field

$\partial_{\alpha} f^{\alpha \beta}=\mu_{0} j^{\beta}-\partial_{\alpha} F^{\alpha \beta}$. 
The energy-momentum density tensor $\theta_{\tau}^{\alpha}\left[\mathrm{Jm}^{-3}\right]$ for the $\mathrm{dBP}$ photon is obtained after a lengthy computation and is given by

$$
\begin{aligned}
\theta_{\tau}^{\alpha}= & \frac{1}{\mu_{0}}\left[f^{\alpha \beta} f_{\beta \tau}+\mathcal{M}^{2} a^{\alpha} a_{\tau}+\mathcal{M}^{2} A^{\alpha} a_{\tau}\right. \\
& \left.+\delta_{\tau}^{\alpha}\left(\frac{1}{4} f^{2}-\frac{1}{2} \mathcal{M}^{2} a^{2}-\mathcal{M}^{2} A^{\beta} a_{\beta}\right)\right] .
\end{aligned}
$$

For $\vec{E}, \vec{B}$, the electric and magnetic fields of the background, $\vec{e}, \vec{b}$, the electric and magnetic fields of the photon, $\Phi, \vec{A}$, scalar and vector potentials of the background, and $\phi, \vec{a}$, scalar and vector potentials of the photon, we make explicit first the energy density $\left[\mathrm{Jm}^{-3}\right]$

$$
\begin{aligned}
& \theta_{0}^{0}=\frac{1}{2}\left\{\epsilon_{0} e^{2}+\frac{1}{\mu_{0}} b^{2}\right. \\
& \left.+\mathcal{M}^{2}\left[\left(\epsilon_{0} \phi^{2}+\frac{1}{\mu_{0}} a^{2}\right)+2 \frac{\vec{A} \cdot \vec{a}}{\mu_{0}}\right]\right\},
\end{aligned}
$$

where $\epsilon_{0}=8.854 \times 10^{-12}\left[\mathrm{~A}^{2} \mathrm{~s}^{4} \mathrm{~kg}^{-1} \mathrm{~m}^{-3}\right]$ is the electric permittivity, and second we make explicit the generalised Poynting density vector $\left[\mathrm{Jm}^{-3}\right]$

$\theta_{0}^{i}=\frac{1}{\mu_{0} c}\left[\vec{e} \times\left.\vec{b}\right|_{i}+\mathcal{M}^{2} \phi\left(a_{i}+A_{i}\right)\right]$.

The energy-momentum density tensor variation $\partial_{\alpha} \theta_{\tau}^{\alpha}$ $\left[\mathrm{Jm}^{-4}\right]$ is given by

$$
\partial_{\alpha} \theta_{\tau}^{\alpha}=\underbrace{j^{\alpha} f_{\alpha \tau}-\frac{1}{\mu_{0}}\left(\partial_{\alpha} F^{\alpha \beta}\right) f_{\beta \tau}}_{\text {Maxwellian terms }} \underbrace{+\frac{1}{\mu_{0}} \mathcal{M}^{2}\left(\partial_{\tau} A^{\beta}\right) a_{\beta}}_{\text {de Broglie-Proca term }},
$$

which, after multiplication by $c$, in the explicit form becomes $\left[\mathrm{Jm}^{-2} \mathrm{~s}^{-1}\right]$

$$
\begin{aligned}
c & \left(\partial_{0} \theta_{0}^{0}+\partial_{i} \theta_{0}^{i}\right) \\
= & \partial_{t} \theta_{0}^{0}+c \partial_{i} \theta_{0}^{i} \\
= & -\vec{j} \cdot \vec{e}-\epsilon_{0}\left(\partial_{t} \vec{E}\right) \cdot \vec{e}+\frac{1}{\mu_{0}}(\nabla \times \vec{B}) \cdot \vec{e} \\
& -\mathcal{M}^{2}\left[\epsilon_{0}\left(\partial_{t} \Phi\right) \phi-\frac{1}{\mu_{0}}\left(\partial_{t} \vec{A}\right) \cdot \vec{a}\right] .
\end{aligned}
$$

In conclusion, the energy-momentum density tensor of the dBP photon is not conserved. In addition to the Maxwellian terms, the mass couples with the background potential time-derivative.

\section{Light propagation in the SME}

\subsection{Non-conservation}

In contrast to the LSV vector, the LSV tensor does not violate CPT. The frequency LSV shift that we shall be dealing with here is an observable of CPT violation, since it depends on the vector and tensor formulations. Incidentally, we shall see that the leading term is $k_{0}^{\mathrm{AF}}$ which violates CPT.

Indicating by the symbol $*$ the dual field, the photon energy-momentum density tensor $\theta_{\tau}^{\alpha}\left[\mathrm{Jm}^{-3}\right]$ is $[27,35]$

$$
\begin{aligned}
\theta_{\tau}^{\alpha}= & \frac{1}{\mu_{0}}\left(f^{\alpha \nu} f_{v \tau}+\frac{1}{4} \delta_{\tau}^{\alpha} f^{2}-\frac{1}{2} k_{\tau}^{\mathrm{AF} *} f^{\alpha \nu} a_{\nu}\right. \\
& \left.+k_{\mathrm{F}}^{\alpha \nu \kappa \lambda} f_{\kappa \lambda} f_{\nu \tau}+\frac{1}{4} \delta_{\tau}^{\alpha} k_{\mathrm{F}}^{\kappa \lambda \nu \beta} f_{\kappa \lambda} f_{\nu \beta}\right) .
\end{aligned}
$$

We render explicit the energy density $\left[\mathrm{Jm}^{-3}\right]$

$$
\begin{aligned}
\theta_{0}^{0}= & \frac{1}{2}\left[\epsilon_{0}\left(\delta_{i j}-2 \chi_{i j}\right) e_{i} e_{j}\right. \\
& \left.+\frac{1}{\mu_{0}}\left(\delta_{i j}+2 \zeta_{i j}\right) b_{i} b_{j}-k_{0}^{\mathrm{AF}} \vec{b} \cdot \vec{a}\right],
\end{aligned}
$$

where $k_{0}^{\mathrm{AF}}$ represents the time component of the breaking vector and the $k_{\mathrm{F}}^{\alpha \nu \kappa \lambda}$ tensor is decomposed as

$k_{\mathrm{F}}^{0 i 0 j}=\chi_{i j}=\chi_{j i}$,

$k_{\mathrm{F}}^{0 i j k}=\epsilon_{i k l} \xi_{i l}$,

$k_{\mathrm{F}}^{i j k l}=\epsilon_{i j m} \epsilon_{k l n} \zeta_{m n} ;$

$\chi_{i j}$ and $\zeta_{m, n}$ are symmetric in the indexes, $\xi_{i l}$ does not have symmetry properties; $\chi_{i j}$ and $\zeta_{m, n}$ have 6 components each, while $\xi_{i l}$ has 9 , totalling the 21 components of $k_{\mathrm{F}}^{\alpha \nu \kappa \lambda}$. We now render explicit the generalised Poynting density vector $\left[\mathrm{Jm}^{-3}\right]$

$$
\begin{aligned}
\theta_{0}^{i}= & \frac{1}{\mu_{0} c} \vec{e} \times\left.\vec{b}\right|_{i}-2 \frac{1}{\mu_{0}}\left(\frac{1}{c} \epsilon_{i j l} \xi_{k l} e_{k} b_{j}+\epsilon_{i j k} \zeta_{k l} b_{j} b_{l}\right) \\
& -\frac{1}{2} k_{0}^{\mathrm{AF}}\left(\epsilon \phi e_{i}-\frac{1}{\mu_{0} c} \vec{a} \times\left.\vec{e}\right|_{i}\right) .
\end{aligned}
$$

The energy-momentum density tensor variation $\partial_{\alpha} \theta_{\tau}^{\alpha}$ $\left[\mathrm{Jm}^{-4}\right]$ is given by

$$
\begin{aligned}
\partial_{\alpha} \theta_{\tau}^{\alpha}= & \underbrace{j^{v} f_{v \tau}-\frac{1}{\mu_{0}}\left(\partial_{\alpha} F^{\alpha \nu}\right) f_{v \tau}}_{\text {Maxwellian terms }} \\
& -\frac{1}{\mu_{0}}[\underbrace{\frac{1}{2}\left(\partial_{\alpha} k_{\tau}^{\mathrm{AF}}\right) * f^{\alpha \nu} a_{v}-\frac{1}{4}\left(\partial_{\tau} k_{\mathrm{F}}^{\alpha \nu \kappa \lambda}\right) f_{\alpha \nu} f_{\kappa \lambda}}_{\text {EM background independent terms }}
\end{aligned}
$$




$$
+\underbrace{\partial_{\alpha}\left(k_{\mathrm{F}}^{\alpha \nu \kappa \lambda} F_{\kappa \lambda}\right) f_{\nu \tau}}_{\text {non-constant term }}+\underbrace{k_{\alpha}^{\mathrm{AF} *} F^{\alpha \nu} f_{\nu \tau}}_{\text {constant term }}] .
$$

After multiplication by $c$, the energy-momentum density tensor variation in the explicit form becomes $\left[\mathrm{Jm}^{-2} \mathrm{~s}^{-1}\right]$

$$
\begin{aligned}
c\left(\partial_{0} \theta_{0}^{0}+\partial_{i} \theta_{0}^{i}\right) \\
=\partial_{t} \theta_{0}^{0}+c \partial_{i} \theta_{0}^{i} \\
=-\vec{j} \cdot \vec{e}-\epsilon_{0}\left(\partial_{t} \vec{E}\right) \cdot \vec{e}+\frac{1}{\mu_{0}}(\nabla \times \vec{B}) \cdot \vec{e} \\
\quad-\frac{1}{2} \frac{1}{\mu_{0} c}\left\{\left(\partial_{t} k_{0}^{\mathrm{AF}}\right) \vec{e} \cdot \vec{a}-\left(\nabla k_{0}^{\mathrm{AF}}\right) \cdot[\phi \vec{e}+c(\vec{a} \times \vec{b})]\right\} \\
+\epsilon_{0}\left(\partial_{t} \chi_{i j}\right) e_{i} e_{j}-2 \frac{1}{\mu_{0} c}\left(\partial_{t} \xi_{i j}\right) e_{i} b_{j}+4 \frac{1}{\mu_{0}}\left(\partial_{t} \zeta_{i j}\right) b_{i} b_{j} \\
\quad+2 \epsilon_{0} \partial_{t}\left(\chi_{i j} E_{j}\right) \cdot e_{i}-2 \frac{1}{\mu_{0} c} \partial_{t}\left(\xi_{i j} B_{j}\right) \cdot e_{i} \\
+\frac{1}{\mu_{0} c} \vec{e} \times\left.\nabla\right|_{i} \cdot\left(2 \xi_{j i} E_{j}+c \zeta_{i j} B_{j}\right) \\
\quad-\frac{1}{\mu_{0} c}\left[\vec{k} \mathrm{AF} \cdot(\vec{E} \times \vec{e})+c k_{0}^{\mathrm{AF}} \vec{B} \cdot \vec{e}\right]
\end{aligned}
$$

The energy-momentum density tensor variation is due to the following contributions:

- Maxwellian, LSV-independent terms that we have seen in Sect. 2.

There are three massive contributions (though not all the components are mass dependent).

- EM background independent terms implying that the energy-momentum density flux is not conserved in the absence of EM fields, if the LSV fields are space-time dependent. This is really a distinctive feature of the SME.

- An LSV and EM space-time dependent term.

- A constant term coming solely from the CPT-odd handedness represented by the Carroll-Field-Jackiw (CFJ) electrodynamics [59]. Its action entails a non-constant 4potential, for a constant EM background and a constant $k_{\mathrm{AF}}$. Indeed, there is an explicit $x^{\alpha}$ coordinate dependence at the level of the Lagrangian, exactly as in the dBP theory.

The breaking tensor $k_{\mathrm{F}}$ appears either under a derivative or coupled to a derivative of the EM background.

The above contributions determine the energy-momentum non-conservation, according to the Noether theorem (symmetry breaking). Put another way, the photon exchanges energy with the LSV and EM fields.

Comparing the non-conservation in $\mathrm{dBP}$ and SME cases, the former is relativistic and respects LoSy, while the latter is gauge-invariant. The advantage of the latter lies in tracing the origin of the mass in the LSV vacuum energy. In both cases a modification of the SM is necessary.
Table 1 Upper limits of the LSV parameters, in SI units: ${ }^{1}$ Rotation in the polarisation of light in resonant cavities [29]. ${ }^{2}$ Astrophysical observations [28]. The latter estimate is close to the Heisenberg limit on the smallest measurable energy or mass or length for a given time, $t$, set equal to the age of the universe. A full table of values was shown in [35]

\begin{tabular}{ll}
\hline$k_{0}^{\mathrm{AF} 1}$ & $5.1 \times 10^{-10} \mathrm{~m}^{-1}$ \\
$k_{0}^{\mathrm{AF} 2}$ & $5.1 \times 10^{-28} \mathrm{~m}^{-1}$ \\
\hline
\end{tabular}

\subsubsection{Origin of the non-conservation}

An estimate of the energy change that light would undergo was given [35]. The wave-particle correspondence, even for a single photon [66], leads us to consider that the light-wave energy non-conservation is translated into photon energy variation and thereby into a red or a blue shift. The energy variations, if there are losses, would translate into frequency damping.

According to [67], the LoSy breaking 4-vector, $k_{\mathrm{AF}}$, and the rank-4 tensor, $k_{\mathrm{F}}$, correspond, respectively, to the vacuum condensation of a vector and a tensor field in the context of string models. They describe part of the vacuum structure, which appears in the form of space-time anisotropies. Therefore, their presence on the right-hand side of Eq. (17) reveals that vacuum effects are responsible for the energy variation of light waves, which, in turn, correspond to a photon frequency shift. In plain terms, vacuum anisotropies are the really responsible for changing the frequency of light emitted by astrophysical structures.

\subsection{Sizing the LSV frequency shift}

In Eq. (17), we may neglect the tensorial perturbation in the frame of Super-Symmetry (SuSy) since it is less likely to condense than the CFJ vectorial perturbation $[35,68,69]$. Independently of SuSy, the $k_{\mathrm{F}}$ term can be neglected since it is quadratic in the field strength and in frequency. The CFJ term instead contains a single derivative and is linear in the frequency. Thus, for optical frequencies of the SNeIA, the $k_{\mathrm{AF}}$ is the dominant contribution.

Excluding the space-time dependent components of the (inter-) galactic magnetic and LSV fields, only one contribution, the last term in Eqs. $(17,18)$, survives. Finally, excluding external currents and large scale electric fields, an estimate was given along the line of sight observer-source for this term [35],

$\partial_{\alpha} \theta_{0}^{\alpha} \approx-\frac{1}{\mu_{0}} k_{0}^{\mathrm{AF} *} F^{0 i} f_{i 0}$,

where ${ }^{*} F^{0 i}$ are the magnetic components of the dual EM background tensor field and $f_{i 0}$ are the electric components of the EM photon tensor field. 
There is no relation between the $k_{0}^{\mathrm{AF}}$ and the photon mass ${ }^{1}$, though there are obviously massive terms in Eq. (17).

Assuming that the energy variation of a light-wave corresponds to a frequency shift for a photon, we converted the energy variation given by Eq. (19) into $\Delta v, v$ being the photon frequency. For $v=486 \mathrm{THz}$ [35]

$|\Delta v|_{\mathrm{LSV}}^{486 \mathrm{THz}} \approx 3.6 \times 10^{47} B f_{i 0} t_{\mathrm{LB}} k_{0}^{\mathrm{AF}} \varrho$,

where $t_{\mathrm{LB}}$ is the look-back time and $\varrho$ is an arbitrary attenuation parameter that takes into account that the magnetic fields (host galaxy, intergalactic, Milky Way) estimated at $B=5 \times 10^{-10}-5 \times 10^{-9} \mathrm{~T}$ each, passed through by the photon, probably have different orientations and thus compensate for each other. We have not considered any relevant magnetic field at the source.

For a numerical estimate of Eq. (22), let us consider a source at $z=0.5, t_{\mathrm{LB}}=1.57 \times 10^{17} \mathrm{~s}, f_{i 0}=3.79 \times$ $10^{-9} \mathrm{~V} \mathrm{~s} \mathrm{~m}^{-2}$, an average $B=2.75 \times 10^{-9} \mathrm{~T}$. We wish to determine what the maximum level of $z_{\text {LSV }}$ could be.

For $k_{0}^{\mathrm{AF}}$ the laboratory and astrophysical upper limits are $5.1 \times 10^{-10} \mathrm{~m}^{-1}$ and $5.1 \times 10^{-28} \mathrm{~m}^{-1}$, respectively $[28,29]$, see Table 1 .

This leads to a value of the order of $10 \%$ of the total $z$, for $\varrho \approx 8 \times 10^{-26}$ and $\varrho \approx 8 \times 10^{-8}$, respectively. These estimates are arbitrary and just show that they can recover the largest frequency shifts in agreement with observational data from SNeIa. Nevertheless, with different assumptions on $\varrho$ and $k_{0}^{\mathrm{AF}}$, we can get considerably smaller percentages of the rate $z_{\mathrm{LSV}} / z$ that are still in agreement with data.

Indeed, it is important to note that the single $z_{\mathrm{LSV}}$ shift from a single SNIa may be small or large, red or blue, depending on the amplitude and orientations of the LSV (vector or tensor) and of the EM fields (host galaxy, intergalactic medium, Milky Way), and obviously the distance of the source. In any case, the final $z_{\mathrm{LSV}}$ is the result of accumulated shifts, both red and blue, encountered along the path.

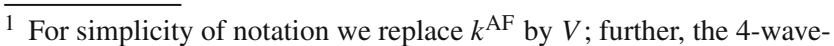
vector is $k^{\mu}=\left(\frac{\omega}{c}, \vec{k}\right)$, where $k^{2}=\left(\frac{\omega^{2}}{c^{2}}-\vec{k}^{2}\right)$. Equations (3) in [26] or (9) in [27] confirm the CFJ dispersion relation [59]; that is,

$$
\begin{aligned}
& \left(k^{\mu} k_{\mu}\right)^{2}+\left(V^{\mu} V_{\mu}\right)\left(k^{v} k_{\nu}\right)-\left(V^{\mu} k_{\mu}\right)^{2} \\
& =\left[\left(\frac{\omega}{c}\right)^{2}-\vec{k}^{2}\right]^{2}+\left(V_{0}^{2}-\vec{V}^{2}\right)\left[\left(\frac{\omega}{c}\right)^{2}-\vec{k}^{2}\right]-\left(V_{0} \frac{\omega}{c}-\vec{V} \vec{k}\right)^{2}=0 .
\end{aligned}
$$

For a massive photon, the rest mass is computed in the rest frame of the photon, $\vec{k}=0$, rendering Eq. (20) as

$\left(\frac{\omega}{c}\right)^{4}+\left(V_{0}^{2}-\vec{V}^{2}\right)\left(\frac{\omega}{c}\right)^{2}-\left(V_{0} \frac{\omega}{c}\right)^{2}=0$.

Equation (21) has two solutions: one non-massive for $\omega=0$ (since gauge symmetry is not broken in CFJ formalism), the other for $\omega=c|\vec{V}|$. In conclusion, the time component of the CFJ perturbation vector does not contribute to the photon mass.
We have set upper limits starting from the physics; in the following, we shall set upper limits from the cosmological data in answer to the question of what range of values of $z_{\text {LSV }}$ can be accommodated by cosmology. In following sections, we shall refer to a generic $z_{\text {LSV }}$ given by all the terms in Eq. (17).

\subsection{Recasting $z$}

The existence of a photon frequency shift not due to the relative motion of the source and the observer belongs to the realm of physics. The role of such shifts in cosmology can attain three levels: replace totally or accompany to a certain degree the expansion shift. In the case of total replacement, we would return to the conception of a static universe, which still passes some tests but fails many others [2,3]. At the other extreme, such a shift could be marginal for cosmology, but nevertheless be of relevance for fundamental physics.

Here, we explore an intermediate option for which the static shift $z_{\mathrm{LSV}}$ is superposed on the expansion shift $z_{C}$.

After recalling that the definition of $z=\Delta v / v_{o}$, where $\Delta v=v_{\mathrm{e}}-v_{o}$ is the difference between the observed $v_{o}$ and emitted $\nu_{\mathrm{e}}$ frequencies, or else $z=\Delta \lambda / \lambda_{\mathrm{e}}$ for the wavelengths, we pose the following conjecture: expansion causes $\lambda_{\mathrm{e}}$ to stretch to $\lambda_{\mathrm{c}}$; that is, $\lambda_{\mathrm{c}}=\left(1+z_{\mathrm{C}}\right) \lambda_{\mathrm{e}}$.

The wavelength $\lambda_{\mathrm{c}}$ could be further stretched or shrunk for the LSV shift to $\lambda_{\mathrm{o}}=\left(1+z_{\mathrm{LSV}}\right) \lambda_{\mathrm{c}}=\left(1+z_{\mathrm{LSV}}\right)\left(1+z_{\mathrm{C}}\right) \lambda_{\mathrm{e}}$. But since $\lambda_{\mathrm{o}}=(1+z) \lambda_{\mathrm{e}}$, we have $1+z=\left(1+z_{\mathrm{C}}\right)\left(1+z_{\mathrm{LSV}}\right)$; thus

$z=z_{\mathrm{C}}+z_{\mathrm{LSV}}+z_{\mathrm{C}} z_{\mathrm{LSV}}:=z_{\mathrm{0}}$

where $z_{\mathrm{o}}$ is the spectroscopically or photometrically observed $z$. The second order is non-negligible for larger $z_{\mathrm{C}}$.

We model the behaviour of $z_{\text {LSV }}$ with distance in three ${ }^{2}$ different ways, Table 2, according to whether the frequency variation is proportional to

- the instantaneous frequency and the distance,

- the emitted frequency and the distance, or

- just the distance.

The $z_{\mathrm{C}}$ shift stems from the expansion of the universe, whereas the $z_{\text {LSV }}$ would occur also along a static distance $r$. The $k_{i}$ parameters can take either positive (frequency increase) or negative (frequency decrease) values.

\footnotetext{
${ }^{2}$ We refrain form considering a fourth option proportional to the observed frequency and the distance.
} 
Table 2 LSV shift types. In the first column, the frequency variation is proportional to the instantaneous frequency and the distance; in the second to the emitted frequency and the distance; in the third only to the distance; $k_{1,2}$ have the dimensions of $\mathrm{Mpc}^{-1}, k_{3}$ of $\mathrm{Mpc}^{-1} \mathrm{~s}^{-1}$. The positiveness of the distance $r$ constraints the shifts, see forth row

\begin{tabular}{llll}
\hline Type & 1 & 2 & 3 \\
\hline$d v$ & $k_{1} v d r$ & $k_{2} v_{\mathrm{e}} d r$ & $k_{3} d r$ \\
$v_{\mathrm{O}}$ & $v_{\mathrm{e}} \exp ^{k_{1} r}$ & $v_{\mathrm{e}}\left(1+k_{2} r\right)$ & $v_{\mathrm{e}}+k_{3} r$ \\
$z_{\mathrm{LSV}}$ & $\exp ^{-k_{1} r}-1$ & $-\frac{k_{2} r}{1+k_{2} r}$ & $-\frac{k_{3} r}{v_{e}+k_{3} r}$ \\
$r$ & $-\frac{\ln \left(1+z_{\mathrm{LSV}}\right)}{k_{1}}$ & $-\frac{z_{\mathrm{LSV}}}{k_{2}\left(1+z_{\text {LSV }}\right)}$ & $-\frac{v_{\mathrm{e}} z_{\text {LSV }}}{k_{3}\left(1+z_{\text {LSV }}\right.}$ \\
$r>0$ & $z_{\text {LSV }}>0$ for $k_{1}<0$ & $z_{\text {LSV }}<-1$ or $z_{\text {LSV }}>0$ for $k_{2}<0$ & $z_{\text {LSV }}<-1$ or $z_{\text {LSV }}>0$ for $k_{3}<0$ \\
& $-1<z_{\text {LSV }}<0$ for $k_{1}>0$ & $-1<z_{\text {LSV }}<0$ for $k_{2}>0$ & $-1<z_{\text {LSV }}<0$ for $k_{3}>0$ \\
\hline
\end{tabular}

\section{The LSV frequency shift and dark energy}

\subsection{Supernovae: luminosity and red shift distances}

A greater than expected SNIa luminosity distance $d_{\mathrm{L}}$ for a given red shift led to the proposal of dark energy in order to reach consistency with the data $[36,37]$. We pursue the same consistency by using Eq. (23) instead.

We do not intend in this paper to propose a fully fledged alternative cosmology, but solely to take the first steps towards the third option presented in Sect. 1. In the following we do not intend to state strict conclusions for cosmology; instead, we limit ourselves to exploring whether existing data can accommodate our reinterpretation; namely, the recasting of $z$ through the additional non-expansion related shift. For this exploration, we pick a popular cosmology simulator $[70,71]$ and draw from it our initial considerations.

The values that $z_{\mathrm{C}}$ should assume for a fixed $d_{\mathrm{L}}$ are computed for two values $(0.72$ and 0$)$ of $\Omega_{\Lambda}$ energy density and three values $(0.7,0.67,0.74)$ of $h$ in Table 3 . We find that a small percentage correction of the red shift (from 0.01 up to $10 \%$, a range we have proven feasible in Sect. 3 ) allows us to recover the same luminosity distances. The explored range of $z$ values for SNeIa is (0-2). The most distant SNIa is at $z=1.914$ [72]. Inverting Eq. (23), we get

$z_{\mathrm{C}}=\frac{z-z_{\mathrm{LSV}}}{1+z_{\mathrm{LSV}}}$

If the $z_{\text {LSV }}$ shift is blue, and thus negative, the photon gains energy, which implies that the cosmological $z_{\mathrm{C}}$ is higher than the observed $z$. If red, and therefore positive, the photon dissipates energy along its path, which implies that the cosmological $z_{\mathrm{C}}$ is smaller than the observed $z$.

From a first glance at Table 3, it appears that in most cases adding a negative blue $z_{\mathrm{LSV}}$ shift to the cosmological $z_{\mathrm{C}}$ is sufficient for agreement between $d_{\mathrm{L}}$ and $z$. Instead, the red shifts appear for $h=0.67$ and small values of $z$. It is also evident that for higher $h$ values a larger $z$ recasting is necessary, and that the shift is bluer. Patently, these are macroscopic and averaged indications. The next step necessitates picking each SNIa from a catalogue and evaluating the associated $z_{\text {LSV }}$.

Preliminary simulations show that $z_{\text {LSV }}$ turns into red or blue shifts, depending also on other cosmological parameters, e.g., the value of matter density $\Omega_{\mathrm{m}}$ or of the curvature $\Omega_{\mathrm{k}}$, and on the behaviour of $z_{\mathrm{LSV}}$ with frequency and distance, Table 2.

At the bottom of Table 3, we have added two lines for $z=4$ and $z=11$ to gauge the behaviour of $z \mathrm{LSV}$ at large $z$ : the most distant superluminous $\mathrm{SN}$ is at $z=3.8893$ [73], and the most distant galaxy is at $z=11.09$ [74].

The data in Table 3 can be commented on by momentarily adopting the usual terminology of accelerated expansion. The acceleration is not observable in our immediate neighbourhood; it would start to be noticeable further out and increase up to a maximum at $z \approx 0.5$. After this threshold value, the acceleration would decrease and for approximately $z \geq 4$, the so-called turning point, it would change sign corresponding to the deceleration phase associated with the older universe.

How to reinterpret the above through a recast $z$ ? Far out, for approximately $z \geq 4, z_{\mathrm{LSV}}$ becomes definitely (and more and more) red. Since a red $z_{\text {LSV }}$ shift corresponds to dissipation, we feel comforted by this easy interpretation for remote distances.

Instead, the major difficulty is explaining why $z_{\mathrm{LSV}}$ starts from a small (blue or red) value in our immediate neighbourhood and increases up to a (blue) maximum for $z \approx 0.5$. This is maybe due to our location in a local void, or other manifestations of inhomogeneities or anisotropies. But before embarking in this sort of argument, the controversy on the data supporting dark energy $[44-57,75]$ should be first of all settled.

\subsubsection{Time dilation and Supernovae}

Since the SME induces an effective mass to the photon $[26,27]$, we are dealing with massive photons. Blondin et al. [76] compared several spectra from the more distant SNeIa 


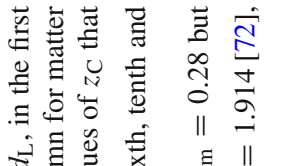

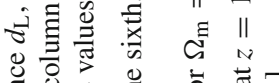

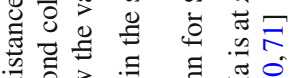

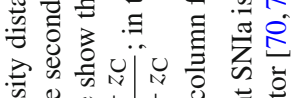

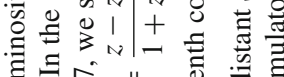

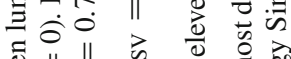

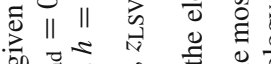

का चुच

bo $\|$ व

言 11 में

ठष

总

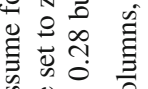

वे

菅 总

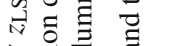

矛密范

这表

ซิ ฮี

结思导

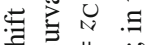

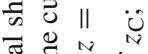

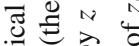

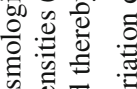

过 항

¿

茊

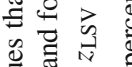

记

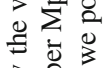

32

क्षे है

등

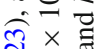

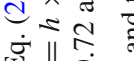

ज्ञ

!! 它我

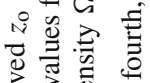

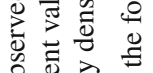

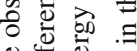

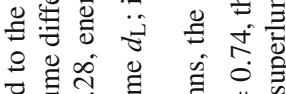

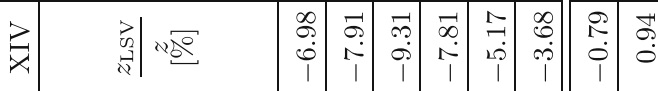

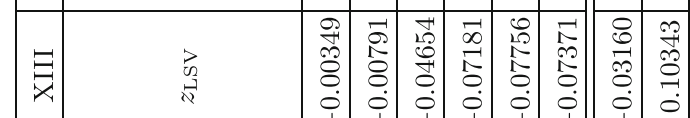

两

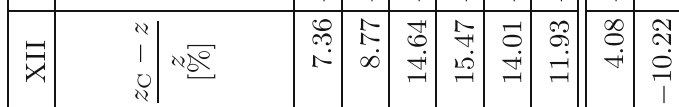

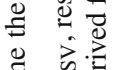

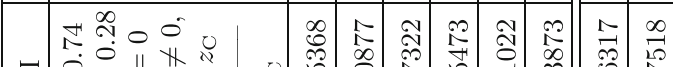

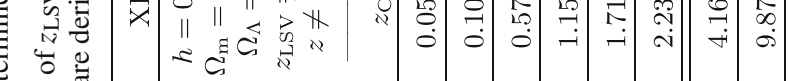

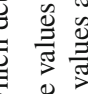

㖊

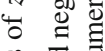

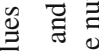

疍

$\cong$ 要守

ธิ

II

$\therefore$ क्षे

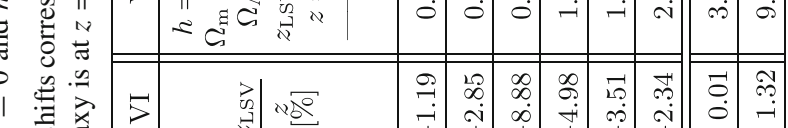

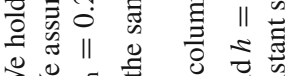

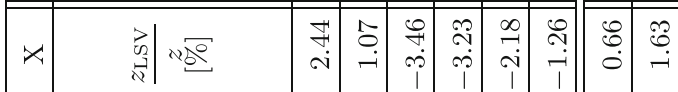

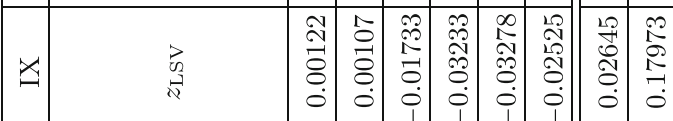

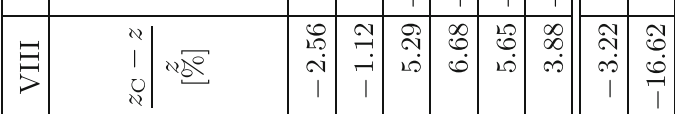

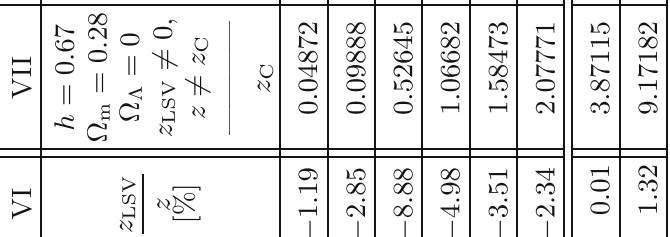

C 名

吾范

क $\overrightarrow{0}:=$

$1 \mid$

c

政

吾

च

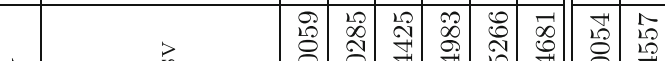

可

势

i|

需

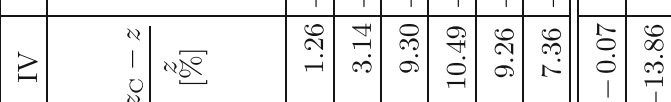

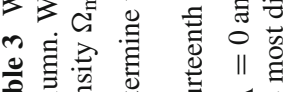

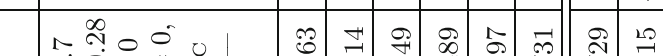

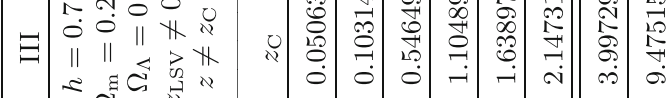

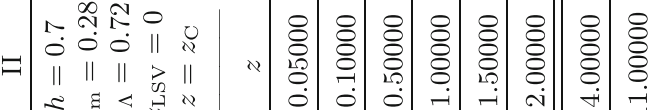

ए

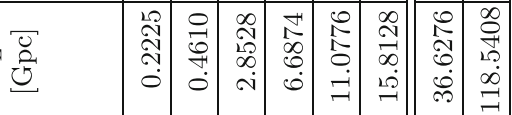

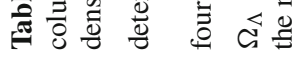


with those of nearer ones and found that the more distant explosions took longer to unfold. They pointed out that time dilation in SNeIa cannot be attributed to 'tired light'. Let us discuss not only this statement in the context of this paper, but also our assumptions on reinterpreting $z$ by examining three related issues.

Firstly, just attributing mass to a photon does not determine per se a decaying frequency, a feature of the 'tired light'. Indeed, massive photon propagation obeys the $\mathrm{dBP}$ equation which, in the absence of an EM background, is written as [23]

$$
\left[\square+\left(\frac{m_{\gamma} c}{\hbar}\right)^{2}\right] a^{\alpha}=0 .
$$

Equation (25) does not entail frequency decay, unless the mass has an imaginary component and there is a singular source term on the right-hand side $[77,78]$. For violating photon energy conservation, apart an external current term, there must be an EM background field, Eq. (9).

Secondly, the energy variation computed here occurs regardlessly of the expansion. The associated shift has been named $z_{\text {LSV }}$ and evaluated to be at most $10 \%$ of the total $z$. It is legitimate to ask whether there is any impact on time dilation due to this reinterpretation of $z$.

In an expanding universe, the ratio of the observed frequency to that emitted by a distant object or the observed rate of any time variation in the intensity of the emitted radiation will be proportional to

$\frac{1}{1+z}$.

Blondin et al. [76] estimated $(1+z)^{b}$ as $b=0.97 \pm 0.10$, $b=1$ being the value predicted by the Friedmann-LemaitreRobertson-Walker cosmology. A variation of $10 \%$ for $b$ is tantamount to a variation of the same order for $z$, as the Taylor expansion shows

$\frac{1}{(1+z)^{b}} \simeq 1-b z+\frac{1}{2}\left(b+b^{2}\right) z^{2}$.

Therefore, supposing the total $z$ as the sum of an expansion related $z_{\mathrm{C}}$ and a static $z_{\mathrm{LSV}}$ is compatible with the findings in [76].

Thirdly, the massive photon group velocity differs from $c$ by a quantity proportional to the inverse of the frequency squared [9-11,23]. Such a dependence has been analysed recently with the signals from Fast Radio Bursts (FRBs) [7988].

It appears that the SNIa spectra often shift from higher, $f_{\mathrm{h}} \approx 8 \times 10^{14} \mathrm{~Hz}$, to lower, $f_{1} \approx 4 \times 10^{14} \mathrm{~Hz}$, optical frequencies during the burst [76]. Photons emitted at the end will therefore take more time to reach the observer than those at start. This delay would occur even when the source is static, mimicking time dilation. For a source at distance $d$, the difference in arrival times is to be added to the burst duration. In SI units, the difference in arrival times is [82]

$$
\Delta t=\frac{d c^{3} m_{\gamma}^{2}}{8 \hbar^{2} \pi^{2}}\left(\frac{1}{f_{1}^{2}}-\frac{1}{f_{\mathrm{h}}^{2}}\right) \simeq \frac{d}{c}\left(\frac{1}{f_{1}^{2}}-\frac{1}{f_{\mathrm{h}}^{2}}\right) 10^{100} m_{\gamma}^{2} .
$$

Inserting the official upper limit on photon mass $10^{-54}$ $\mathrm{kg}, d=1.4 \times 10^{9}$ light-years (approximately corresponding to $z=0.1), \Delta t$ is in the order of $10^{-21} \mathrm{~s}$. In an expanding universe, Eq. (28) becomes $[79,81]$

$\Delta t=\frac{1}{H}\left(\frac{1}{f_{1}^{2}}-\frac{1}{f_{\mathrm{h}}^{2}}\right) 10^{100} m_{\gamma}^{2} H_{\gamma}$,

where

$H_{\gamma}=\int_{0}^{z} \frac{\left(1+z^{\prime}\right)^{-2}}{\sqrt{\Omega_{m}\left(1+z^{\prime}\right)^{3}+\Omega_{\Lambda}}} d z^{\prime}$

Equation (30) reduces the outcome of Eq. (28) by a factor 10 for common values of the $\Omega$ densities. Hence, we have shown that although massive photons determine an effect similar to time dilation for not-moving sources or add a static contribution to moving sources, such an effect is utterly marginal for SNeIa.

In conclusion, our assumptions and results are compatible with current literature on SNeIa.

\section{2 $\mathrm{BAO}, \mathrm{CMB}$ and gravitational lensing}

In Sect. 1, we gave a brief account on the debates on (1) the nature of dark energy, (2) the data reliability from SNeIa, $\mathrm{BAO}, \mathrm{CMB}$ and gravitational lensing, and (3) the alternatives to dark energy. The latter two debates are not dominant as there is a prevailing tendency to converge towards the $\Lambda \mathrm{CDM}$ Concordance Model.

Nevertheless, there is an increasing interest in discussing the foundations of common assumptions. A critical analysis of the supposed confirmation of dark energy by the Baryonic Atomic Oscillations (BAO) and by the Cosmic Microwave Background $(\mathrm{CMB})$ has recently appeared $[53,54]$ in which it is shown that that a non-accelerated universe is nicely able to fit low and high red shift data from SNeIa, BAO and the $\mathrm{CMB}$ on the main assumption of SN luminosity dependency on red shift. Now, Kang et al. [75] have found a significant (a 99.5\%) correlation between SN luminosity and stellar population age. It is worth recalling that the CMB can be interpreted differently, possibly without dark energy [89] and in any case outside a canonical flat universe [58].

We have interpreted $z_{\text {LSV }}$ as due to vacuum energy, an effective dark energy of LSV origin, although not producing acceleration of the expansion. We do not dispute the results from $\mathrm{BAO}$ and the $\mathrm{CMB}$ proving the existence of dark energy per se. Our interpretation would be falsified, if there were a strict proof of acceleration $\left(\mathrm{m} / \mathrm{s}^{2}\right)$ with respect to an inertial 
frame. Further, a two-point in time measurement of distances is too small to serve as proof of acceleration since in the order of $10^{-9}$ (observation interval 10 years/age of the universe $10^{10}$ years).

It is worth considering that BAO and CMB data can accommodate our recasting of $z$ due to the uncertainties of spectroscopic and especially photometric measurements. In particular for the CMB, the photons were all emitted at the same time and same distance from us. Thus, any change in the definition of the red shift should not bear huge consequences.

For BAO, the angular size of the sound horizon is measured for the large-scale structure at different red shifts, which allows us to measure $H(z)$, which depends on $\Omega_{\mathrm{m}}$, $\Omega_{\Lambda}$ and other variables. Since $d_{\mathrm{A}}$ (angular distance) is proportional to $d_{\mathrm{L}} /(1+z)^{2}$ and the angular size of an object is inversely proportional to $d_{\mathrm{A}}$, it would not be surprising that the same $z_{\text {LSV }}$ mimicking the dark energy for SNeIa, acts similarly for the BAO peak.

Finally, we tackle gravitational lensing. The classical method used to probe dark energy is to measure the ratio of average tangential shear as a function of the red shift behind the clusters [90]. This ratio depends on the relation between distance and red shift, which is modified by dark energy, or by $z_{\text {LSV }}$.

\section{Discussion of the main results}

We have assumed the total red shift $z$ as a combination of the expansion red shift $z_{\mathrm{C}}$ and of a static, red or blue shift $z_{\mathrm{LSV}}(r)$, $r$ being the travelled distance. The latter shift is due to the energy non-conservation of a photon propagating through EM background fields (host galaxy, intergalactic and Milky Way). Beyond the Maxwellian contributions, if the photon is massive, such propagation may be described within the framework of de Broglie-Proca (dBP) formalism or within the framework of the Lorentz(-Poincaré) Symmetry Violation (LSV) associated with the Standard-Model Extension (SME). In the latter case, the non-conservation stems from the vacuum expectation value of the vector and tensor LSV fields.

Our understanding is that $z_{\mathrm{LSV}}$ is a manifestation of an effective dark energy caused by the expectation values of the vacuum under LSV. Indeed, we suggest that dark energy, i.e. LSV vacuum energy, is not causing an accelerated expansion but a frequency shift.

The single $z_{\mathrm{LSV}}$ shift from a single SNIa may be small or large, red or blue, depending on the orientations of the LSV (vector or tensor) and of the EM fields (host galaxy, intergalactic medium, Milky Way), as well as the distance of the source. In any case, the colour of $z$ LSV is the final output of a series of shifts, both red and blue, encountered along the path.
If the $z_{\text {LSV }}$ shift is blue, thus negative, the photon gains energy; it implies that the real red shift is larger than the measured $z$. If red, thus positive, $z_{\text {LSV }}$ corresponds to dissipation along the photon path; it implies that the real red shift is smaller than the measured $z$.

Recasting $z$, on average, we observe a blue static shift for $z \leq 2$, but a red one in our local universe for smaller values of the Hubble(-Humason)-Lemâitre parameter $(67-74 \mathrm{~km} / \mathrm{s}$ per Mpc), and always red for $z>4$.

The peculiarity of our approach is that a single mechanism could explain all the positions of the SNeIa in the $(\mu, z)$ plan, $\mu$ being the distance modulus, including the outliers. The experimental and observational limits on LSV and magnetic fields are fully compatible with our findings.

\section{Perspectives}

The LSV shift provides a physical explanation of red shift remapping [91-94] and is not limited to the SNIa case. It is naturally suited to explaining recently discovered expansion anisotropies [55-57,95].

In future work, leaving aside massive photons, we will show that a frequency shift is also produced by a generalised non-linear electromagnetism, encompassing the formulations of Born and Infeld, and Euler, (Kockel) and Heisenberg. The calculated limits on $z_{\mathrm{LSV}}$ will be applicable to $z_{\mathrm{NL}}$ for the non-linear electromagnetism.

Apart from an additional shift occurring also in Maxwellian electromagnetism in the presence of a space-time dependent EM field, we will show that departing from Maxwellian electromagnetism in three different directions (classically massive dBP electromagnetism or non-linear electromagnetism or the SME) leads to a common conclusion: the red shift is not only due to expansion. If and by how much such a departure is relevant for cosmology is the real question.

Nineteenth century Maxwellian electromagnetism and the more modern Einsteinian gravitation have been well tested. This has not impeded the proposition of alternative formulations of electromagnetism and gravity. The lack of experimental proof on the dark universe and the successes of general relativity prompt us to revisit astrophysical observations, largely based on light signals, with non-Maxwellian electromagnetism, opening the door to radically new interpretations.

In future explorations, we will have to deal with the comparison of $z_{\text {LSV }}$ with the error on $z$. The analysis of the error on spectroscopic and photometric measurements is destined to become a pivotal issue for cosmology [96-98].

The $z_{\text {LSV }}$ shift considered here is at most $10 \%$ of the $z_{\mathrm{C}}$ expansion shift relative to $H_{0}=70 \mathrm{~km} / \mathrm{s}$ per $\mathrm{Mpc}$ and is thus below $2.3 \times 10^{-19} \Delta v / v$ per $\mathrm{m}$ (units for a static red shift). It would be desirable to test frequency invariance in vacuo [99-102] with a ground or space based interferometer. 
Acknowledgements Discussions with M. A. Lopes Capri, V. E. Rodino Lemes, R. de Oliveira Santos (Rio de Janeiro), M. Benetti and F. Ragosta (Napoli) are acknowledged. SC acknowledges the support of INFN, iniziative specifiche QGSKY and MOONLIGHT2. The authors thank P. Brockill (Milwaukee) for comments and T. Mahoney (La Laguna) for editing support.

Data Availability Statement This manuscript has no associated data or the data will not be deposited. [Authors' comment: All the used data are presented in this work or published in the references.]

Open Access This article is licensed under a Creative Commons Attribution 4.0 International License, which permits use, sharing, adaptation, distribution and reproduction in any medium or format, as long as you give appropriate credit to the original author(s) and the source, provide a link to the Creative Commons licence, and indicate if changes were made. The images or other third party material in this article are included in the article's Creative Commons licence, unless indicated otherwise in a credit line to the material. If material is not included in the article's Creative Commons licence and your intended use is not permitted by statutory regulation or exceeds the permitted use, you will need to obtain permission directly from the copyright holder. To view a copy of this licence, visit http://creativecomm ons.org/licenses/by/4.0/.

Funded by SCOAP ${ }^{3}$.

\section{References}

1. S. Capozziello, T. Prokopec, A.D.A.M. Spallicci, Found. Phys. 47, 709 (2019)

2. M. López-Corredoira, Found. Phys. 47, 711 (2017). arXiv:1701.08720 [astro-ph.CO]

3. M. López-Corredoira, in Proc. 2nd Int. Conf. Cosmology on small scale, edited by M. Křížek, Y. V. Dumin (Inst. Math. Czech Acad. Sciences, Praha, 2018), p. 14, 26-29 September 2018 Praha. arXiv: 1808.09823 [astro-ph.CO]

4. P. A. Zyla and the Particle Data Group, Progr. Theor. Exp. Phys., 083C01 (2020)

5. A. Retinò, A.D.A.M. Spallicci, A. Vaivads, Astropart. Phys. 82, 49 (2016). arXiv:1302.6168 [hep-ph]

6. D.D. Ryutov, Plasma Phys. Contr. Fus. 39, A73 (1997)

7. D.D. Ryutov, Plasma Phys. Contr. Fus. 49, B429 (2007)

8. D.D. Ryutov, Phys. Rev. Lett. 103, 201803 (2009)

9. L. de Broglie, J. Phys. Radium 3, 422 (1922)

10. L. de Broglie, Comptes Rendus Hebd. Séances Acad. Sci. Paris 177, 507 (1923)

11. L. de Broglie, Recherches sur la théorie des quanta (Masson \& $\mathrm{C}^{\mathrm{ie}}$, Paris, 1924), Doctorate thesis (Dir. P. Langevin) Université de Paris, Sorbonne

12. L. de Broglie, Comptes Rendus Hebd. Séances Acad. Sci. Paris 195, 536 (1932)

13. L. de Broglie, Comptes Rendus Hebd. Séances Acad. Sci. Paris 195, 577 (1932)

14. L. de Broglie, Comptes Rendus Hebd. Séances Acad. Sci. Paris 198, 135 (1934)

15. L. de Broglie, Comptes Rendus Hebd. Séances Acad. Sci. Paris 199, 445 (1934)

16. L. de Broglie, Comptes Rendus Hebd. Séances Acad. Sci. Paris 199, 1165 (1934)

17. L. de Broglie, Une Nouvelle Conception de la Lumière, vol. 181 of Actualités Scientifiques et Industrielles (Hermann \& $C^{\text {ie }}$, Paris, 1934)
18. L. de Broglie, Nouvelles Recherches sur la Lumière, vol. 411 of Actualités Scientifiques et Industrielles (Hermann \& $C^{\text {ie }}$, Paris, 1936)

19. A. Proca, Comptes Rendus Hebd. Séances Acad. Sci. Paris 202, 1490 (1936)

20. A. Proca, Comptes Rendus Hebd. Séances Acad. Sci. Paris 203, 709 (1936)

21. A. Proca, J. Phys. Radium 7, 347 (1936)

22. A. Proca, J. Phys. Radium 8, 23 (1937)

23. L. de Broglie, La Mécanique Ondulatoire du Photon. Une Nouvelle Théorie de la Lumière (Hermann \& $\mathrm{C}^{\mathrm{ie}}$, Paris, 1940)

24. D. Colladay, V.A. Kostelecký, Phys. Rev. D 55, 6760 (1997). arXiv:hep-ph/9703464

25. D. Colladay, V.A. Kostelecký, Phys. Rev. D 58, 116002 (1998). arXiv:hep-ph/9809521

26. L. Bonetti, L.R. dos Santos Filho, J.A. Helayël-Neto, A.D.A.M. Spallicci, Phys. Lett. B 764, 203 (2017). arXiv:1607.08786 [hep$\mathrm{ph}]$

27. L. Bonetti, L.R. dos Santos Filho, J.A. Helayël-Neto, A. D. A.M. Spallicci, Eur. Phys. J. C 78, 811 (2018). arXiv:1709.04995 [hepth]

28. V.A. Kostelecký, N. Russell, Rev. Mod. Phys. 83, 11 (2011). arXiv:0801.0287 [hep-ph]

29. Y.M.P. Gomes, P.C. Malta, Phys. Rev. D 94, 025031 (2016). arXiv:1604.01102 [hep-ph]

30. N. Craig, I. Garcia Garcia, J. High Energ. Phys. 11, 067 (2018). arXiv: 1810.05647 [hep-th]

31. J. Alfaro, A. Soto, Phys. Rev. D. 100, 055029 (2019). arXiv:1901.08011 [hep-th]

32. M. Reece, J. High Phys. 7, 181 (2019). arXiv:1808.09966 [hep-th]

33. T.R. Govindarajan, N. Kalyanapuram, Mod. Phys. Lett. 34, 1950009 (2019). arXiv:1810.10209 [hep-th]

34. M.M. Ferreira Jr., J.A. Helayël-Neto, C.M. Reyes, M. Schreck, P.D.S. Silva, Phys. Lett. B 804, 135379 (2020). arXiv:2001.04706 [hep-th]

35. J.A. Helayël-Neto, A.D.A.M. Spallicci, Eur. Phys. J. C 79, 590 (2019). arXiv:1904.11035 [hep-ph]

36. A.G. Riess, A.V. Filippenko, P. Challis, A. Clocchiatti, A. Diercks, P.M. Garnavich, R.L. Gilliland, C.J. Hogan, S. Jha, R.P. Kirshner et al., Astron. J. 116, 1009 (1998). arXiv:astro-ph/9805201

37. S. Perlmutter, G. Aldering, G. Goldhaber, R.A. Knop, P. Nugent, P.G. Castro, S. Deustua, S. Fabbro, A. Goobar, D.E. Groom et al., Astrophys. J. 517, 565 (1999). arXiv:astro-ph/9812133

38. P.J.E. Peebles, B. Ratra, Rev. Mod. Phys. 75, 559 (2003). arXiv:astro-ph/0207347

39. K. Bamba, S. Capozziello, S. Nojiri, S.D. Odintsov, Astrophys. Space Sci. 342, 155 (2012). arXiv:1205.3421 [gr-qc]

40. S. Capozziello, M. Francaviglia, Gen. Rel. Grav. 40, 357 (2008). arXiv:0706.1146 [astro-ph]

41. S. Capozziello, M. De Laurentis, Phys. Rept. 509, 167 (2011). arXiv:1108.6266 [gr-qc]

42. T. Clifton, P.G. Ferreira, A. Padilla, C. Skordis, Phys. Rept. 513, 1 (2012). arXiv:1106.2476 [astro-ph.CO]

43. J.M. Ezquiaga, M. Zumalacárregui, Phys. Rev. Lett. 119, 251304 (2017). arXiv:1710.05901 [astro-ph.CO]

44. R.G. Vishwakarma, J.V. Narlikar, Res. Astron. Astrophys. 10, 1195 (2010). arXiv:1010.5272 [physics.gen-ph]

45. J.T. Nielsen, A. Guffanti, S. Sarkar, Sci. Rep. 6, 35596 (2016). arXiv:1506.01354 [astro-ph.CO]

46. D. Rubin, B. Hayden, Astrophys. J. 833, L30 (2016). arXiv:1610.08972 [astro-ph.CO]

47. B.S. Haridasu, V.V. Luković, R. D'Agostino, N. Vittorio, Astron. Astrophys. 600, L1 (2017). arXiv:1702.08244 [astro-ph.CO]

48. H. Velten, S. Gomes, V.C. Busti, Phys. Rev. D 97, 083516 (2018). arXiv:1801.00114 [astro-ph.CO] 
49. C. Csáki, N. Kaloper, J. Terning, Phys. Rev. Lett. 88, 161302 (2002). arXiv:hep-ph/0111311

50. C. Csáki, N. Kaloper, J. Terning, Phys. Lett. B 535, 33 (2002). arXiv:hep-ph/0112212

51. A. Blanchard, M. Douspis, M. Rowan-Robinson, S. Sarkar, Astron. Astrophys. 412, 35 (2003). arXiv:astro-ph/0304237

52. L.H. Dam, A. Heinesen, D.L. Wiltshire, Mon. Not. Roy. Astron. Soc. 472, 835 (2017). arXiv:1706.07236 [astro-ph.CO]

53. I. Tutusaus, B. Lamine, A. Dupays, L. Blanchard, Astron. Astrophys. 602, A73 (2017). arXiv:1706.05036 [astro-ph.CO]

54. I. Tutusaus, B. Lamine, L. Blanchard, Astron. Astrophys. 625, A15 (2019). arXiv:1803.06197 [astro-ph.CO]

55. J. Colin, R. Mohayaee, M. Rameez, S. Sarkar, Astron. Astrophys. 631, L13 (2019). arXiv:1808.04597 [astro-ph.CO]

56. A. Salehi, H. Farajollahi, M. Motahari, P. Pashamokhtari, M. Yarahmadi, S. Fathi, Int. J. Mod. Phys. 80, 753 (2020). arXiv:1511.04732 [astro-ph.CO]

57. M.E. Moreno-Raya, M. Mollá, A. López-Sánchez, R. Ángel, L. Galbany, J.M. Vílchez, A. Carnero Rosell, I. Domínguez, Astrophys. J. 818, L19 (2016). arXiv:1511.05348 [astro-ph.GA]

58. E. Di Valentino, A. Melchiorri, J. Silk, Nat. Astron. 4, 196 (2019). arXiv:1911.02087 [astro-ph.CO]

59. S.M. Carroll, G.B. Field, R. Jackiw, Phys. Rev. D 41, 1231 (1990)

60. T. Jacobson, D. Mattingly, Phys. Rev. D 64, 024028 (2003). arXiv:gr-qc/0007031

61. R. Potting, in A century of relativity physics, Proc. 28th Spanish Relativity Meeting, ERE 2005, edited by J.D.A.L. Mornas (American Institute of Physics, College Park, 2006), vol. 841, p. 574, 6-10 September 2005 Oviedo. arXiv:0902.0083 [gr-qc]

62. V.A. Kostelecký, N. Russell, J.D. Tasson, Phys. Rev. Lett. 100, 111102 (2008). arXiv:0712.4393 [gr-qc]

63. J.L. Boldo, J.A. Helayël-Neto, L.M. de Moraes, C.A.G. Sasaki, V.J.Vasquez Ottoya, Phys. Lett. B 690, 112 (2010). arXiv:0903.5207 [hep-th]

64. D. Blas, E. Lim, Int. J. Mod. Phys. D 23, 1443009 (2014). arXiv: 1412.4828 [gr-qc]

65. A. Eichhorn, A. Platania, M. Schiffer, Phys. Rev. D 102, 026007 (2020). arXiv:1911.10066 [hep-th]

66. A. Aspect, P. Grangier, Hyperf. Interact. 37, 1 (1987)

67. V.A. Kostelecký, S. Samuel, Phys. Rev. D 39, 683 (1989)

68. H. Belich, L.D. Bernald, P. Gaete, J.A. Helayël-Neto, Eur. Phys. J. C 73, 2632 (2013). arXiv:1303.1108 [hep-th]

69. H. Belich, L.D. Bernald, P. Gaete, J.A. Helayël-Neto, F.J.L. Leal, Eur. Phys. J. C 75, 291 (2015). arXiv:1502.06126 [hep-th]

70. E.L. Wright, Publ. Astron. Soc. Pac. 118, 1711 (2006). arXiv:astro-ph/0609593

71. E.L. Wright, Javascript cosmology calculator (2018). http:// www.astro.ucla.edu/ wright/CosmoCalc.html

72. D.O. Jones, S.A. Rodney, A.G. Riess, B. Mobasher, T. Dahlen, C. McCully, T.F. Frederiksen, S. Casertano, J. Hjorth, C.R. Keeton et al., Astrophys. J. 768, 166 (2013). arXiv:1304.0768 [astroph.CO]

73. J. Cooke, M. Sullivan, A. Gal-Yam, E.J. Barton, R.G. Carlberg, E.V. Ryan-Weber, C. Horst, Y. Omori, C.G. Diaz, Nat. 491, 228 (2012). arXiv:1211.2003 [astro-ph.CO]

74. P.A. Oesch, G. Brammer, P.G. van Dokkum, G.D. Illingworth, R.J. Bouwens, I. Labbe, M. Franx, I. Momcheva, M.L.N. Ashby, G.G. Fazio et al., Astrophys. J. 819, 129 (2016). arXiv:1603.00461 [astro-ph.GA]

75. Y. Kang, Y.-W. Lee, Y.-L. Kim, C. Chung, C.H. Ree, Astrophys. J. 889, 8 (2020). arXiv:1912.04903 [astro-ph.GA]

76. S. Blondin, T.M. Davis, K. Krisciunas, B.P. Schmidt, J. Sollerman, W.M. Wood-Vasey, A.C. Becker, P. Challis, A. Clocchiatti, G. Damke et al., Astrophys. J. 682, 724 (2008). arXiv:0804.3595 [astro-ph]
77. M. Thiounn, Comptes Rendus Hebd. Séances Acad. Sci. Paris B 262, 319 (1960)

78. W. Yourgrau, J.F. Woodward, Acta Phys. Sci. Hungaricae 37, 283 (1974)

79. L. Bonetti, J. Ellis, N.E. Mavromatos, A.S. Sakharov, E.K. Sarkisian-Grinbaum, A.D.A.M. Spallicci, Phys. Lett. B 757, 548 (2016). arXiv: 1602.09135 [astro-ph.HE]

80. X. Wu, S. Zhang, H. Gao, J. Wei, Y. Zou, W. Lei, B. Zhang, Z. Dai, P. Mészáros, Astrophys. J. Lett. 822, L15 (2016). arXiv:1602.07835 [astro-ph.HE]

81. L. Bonetti, J. Ellis, N.E. Mavromatos, A.S. Sakharov, E.K. Sarkisian-Grinbaum, A.D.A.M. Spallicci, Phys. Lett. B 768, 326 (2017). arXiv:1701.03097 [astro-ph.HE]

82. M.J. Bentum, L. Bonetti, A.D.A.M. Spallicci, Adv. Space Res. 59, 736 (2017). arXiv:1607.08820 [astro-ph.IM]

83. L. Shao, B. Zhang, Phys. Rev. D 95, 123010 (2017). arXiv:1705.01278 [hep-ph]

84. J.-J. Wei, E.-K. Zhang, S.-B. Zhang, X.-F. Wu, Res. Astron. Astrophys. 17, 13 (2017). arXiv:1608.07675 [astro-ph.HE]

85. J.-J. Wei, X.-F. Wu, J. Cosm. Astropart. Phys. 7, 045 (2018). arXiv:1803.07298 [astro-ph.HE]

86. Y.-P. Yang, B. Zhang, Astrophys. J. 842, 23 (2017). arXiv:1701.03034 [astro-ph.HE]

87. N. Xing, H. Gao, J. Wei, Z. Li, W. Wang, B. Zhang, X. Wu, P. Mészáros, Astrophys. J. Lett. 882, L13 (2019). arXiv: 1907.00583 [astro-ph.HE]

88. J.-J. Wei, X.-F. Wu, Combined limit on the photon mass with nine localized Fast Radio Bursts, to appear in Res. Astron. Astrophys. arXiv:2006.09680 [astro-ph.HE]

89. M. López-Corredoira, Int. J. Mod. Phys. A 22, 1350032 (2013). arXiv:1303.1375 [astro-ph.CO]

90. A. Heavens, Nucl. Phys. Proc. Suppl. 194, 76 (2009). arXiv:0911.0350 [astro-ph.CO]

91. B.A. Bassett, Y. Fantaye, R. Hložek, C. Sabiu, M. Smith, Observational constraints on redshift remapping. arXiv:1312.2593 [astroph.CO]

92. R. Wojtak, F. Prada, Mon. Not. R. Astron. Soc. 458, 3331 (2016). arXiv:1602.02231 [astro-ph.CO]

93. R. Wojtak, F. Prada, Mon. Not. R. Astron. Soc. 470, 4493 (2017). arXiv: 1610.03599 [astro-ph.CO]

94. S. Tian, Astrophys. J. 846, 90 (2017). arXiv:1709.08322 [astroph.CO]

95. K. Migkas, G. Schellenberger, T.H. Reiprich, F. Pacaud, M.E. Ramos-Ceja, L. Lovisari, Astron. Astrophys. 636, A15 (2020). arXiv:2004.03305 [astro-ph.CO]

96. N. Palanque-Delabrouille, V. Ruhlmann-Kleider, S. Pascal, J. Rich, J. Guy, G. Bazin, P. Astier, C. Balland, S. Basa, R.-G. Carlberg et al., Astron. Astrophys. 514, A63 (2010). arXiv:0911.1629 [astro-ph.CO]

97. J. Calcino, T. Davis, J. Cosm. Astropart. Phys. 1, 38 (2017). arXiv:1610.07695 [astro-ph.CO]

98. T.M. Davis, S.R. Hinton, C. Howlett, J. Calcino, Mon. Not. Roy. Astron. Soc. 490, 2948 (2019). arXiv:1907.12639 [astro-ph.CO]

99. J. Shamir, R. Fox, N. Cim. B 50, 371 (1967)

100. E. Wolf, Phys. Rev. Lett. 56, 1370 (1986)

101. E. Wolf, Nature 326, 363 (1987a)

102. E. Wolf, Phys. Rev. Lett. 58, 2646 (1987b) 